\title{
Combination of early and delayed ischemic postconditioning enhances brain-derived neurotrophic factor production by upregulating the ERK-CREB pathway in rats with focal ischemia
}

\author{
HUI WU，SHAO-FENG YANG，JIONG DAI，YONG-MING QIU，YI-FENG MIAO and XIAO-HUA ZHANG
}

Department of Neurosurgery, Renji Hospital, Shanghai Jiao Tong University School of Medicine, Shanghai 201112, P.R. China

Received July 29, 2014; Accepted April 15, 2015

DOI: $10.3892 / \mathrm{mmr} .2015 .4327$

\begin{abstract}
Ischemic postconditioning, including early and delayed ischemic postconditioning, has been recognized as a simple and promising strategy in the treatment of stroke. However, the effects of the combination of early and delayed ischemic postconditioning, and the mechanisms underlying these effects, remain unclear. The aim of the present study was to determine whether the combination of early and delayed ischemic postconditioning offers greater protection against stroke, and enhances the production of brain-derived neurotrophic factor (BDNF). A combination of early and delayed ischemic postconditioning was established by repeated, transient occlusion and reperfusion of the ipsilateral common carotid artery in a rat model of middle cerebral artery occlusion. Infarct size, motor function, cerebral blood flow and brain edema were then evaluated, in order to confirm the effects of combinative ischemic postconditioning. TUNEL staining was used to analyze the rate of apoptosis of cells in the penumbral area. BDNF, extracellular signal-regulated kinases $1 / 2$ (ERK1/2) and cAMP response element-binding protein (CREB) expression was detected using immunofluorescence staining and western blot analysis. The results of the present study indicated that the combination of early and delayed ischemic postconditioning further reduced the infarct volume, stabilized cerebral blood disturbance and attenuated neuronal apoptosis, compared with either alone. However, combinative postconditioning exerted the same effect on neurological function and brain edema, compared with early or delayed ischemic postconditioning alone. Further investigation indicated that combinative ischemic postconditioning increased the expression of BDNF, and a significantly higher number of BDNF-positive cells was
\end{abstract}

Correspondence to: Professor Xiao-Hua Zhang or Dr Yi-Feng Miao, Department of Neurosurgery, Renji Hospital, Shanghai Jiao Tong University School of Medicine, 2000 Jiangyue Road, Shanghai 201112, P.R. China

E-mail: zxh1969@aliyun.com

E-mail: yifengmwx@hotmail.com

Key words: combination, neuroprotection, brain-derived neurotrophic factor, ischemic postconditioning, focal brain ischemia observed in neurons and astrocytes from the combined group than in the early or delayed groups. Combinative ischemic postconditioning also induced the phosphorylation of ERK1/2 and CREB in the cortex, following focal ischemia. The results of the present study suggest that the combination of early and delayed ischemic postconditioning may further reduce brain ischemic reperfusion injury following focal ischemia, compared with either treatment alone. In addition, it induces the production of BDNF in neurons and astrocytes. Furthermore, the effects of combinative ischemic postconditioning may be mediated by the activation of ERK1/2 and CREB.

\section{Introduction}

Since there are few effective treatments available for acute stroke, further research is required to identify novel strategies with which to treat this condition (1). One such strategy is ischemic postconditioning, which may be induced by repeated cycles of transient reperfusion and re-occlusion of the artery, which is applied immediately after reperfusion (2-4). There are two types of ischemic postconditioning: Early and delayed ischemic postconditioning (5). Rapid postconditioning is induced immediately or a few minutes after reperfusion. Delayed postconditioning may be induced up to 2 days after stroke, however at present 3-6 h is recognized to induce robust neuroprotection after ischemic injury. It has previously been reported that both forms of ischemic postconditioning are able to improve neurological function and reduce infarct volume following focal ischemic injury (6). However, as ischemia is unpredictable, ischemic postconditioning, which may be implemented following a stroke, requires further research and increased clinical attention. Therefore, it may be important to identify additional models of ischemic postconditioning. However, to date, there have been no reports on the effects of the combination of early and delayed ischemic postconditioning, and whether this may provide an increased neuroprotective effect, compared with one type of ischemic postconditioning alone.

Brain-derived neurotrophic factor (BDNF) is a member of the neurotrophin family, which is hypothesized to be one of the most important neurotrophic factors (NTFs) in the central and peripheral nervous systems $(7,8)$. Numerous studies have indicated that NTFs, particularly BDNF, are associated with neuronal development and differentiation, as well as with improvement in neurological function following 
brain injury $(9,10)$. The cAMP response element-binding protein (CREB) is a transcription factor of BDNF (11). In addition, extracellular signal-regulated kinases 1/2 (ERK1/2) is an upstream phosphorylating enzyme of $\operatorname{BDNF}(11,12)$. Therefore, all of these proteins are important for the survival and development of neuronal cells.

The present study aimed to investigate whether early ischemic postconditioning, in combination with delayed ischemic postconditioning, may protect against neuronal injury and behavioral deficits following focal brain ischemia. Furthermore, in order to explore the mechanisms underlying these neuroprotective effects, the effects of ischemic postconditioning on the disturbance of cerebral blood flow (CBF) and brain edema were evaluated. The current study also investigated the role of the ERK1/2-CREB-BDNF pathway in the neuroprotective effects of combinative postconditioning.

\section{Materials and methods}

Animals, surgical procedures and ischemic postconditioning. The experimental protocol of the present study was approved by the ethical committee of the Animal Care and Experimental Committee of the School of Medicine of Shanghai Jiao Tong University (Shanghai, China). A total of 136 age-matched adult male Sprague-Dawley rats (weight, 270-300 g; age, 7-8 weeks), were housed in a temperature- and light-controlled environment with a 14/10-h light/dark cycle. The rats were purchased from the Animal Center of the School of Medicine of Shanghai Jiao Tong University (Shanghai, China). The rats were randomly divided into five groups: Sham-operated (sham; $n=24)$, middle cerebral artery occlusion (MCAO; $\mathrm{n}=30$ ), early ischemic postconditioning (IPe; $\mathrm{n}=28$ ), delayed ischemic postconditioning (IPd; $\mathrm{n}=28$ ) and combinative ischemic postconditioning treatment (IPc; $n=26$ ) groups. Transient cerebral ischemia was induced using MCAO, as previously described (13). Briefly, the rats were anesthetized by inhalation of 5\% isoflurane (Ohio Medical Corporation, Gurnee, IL, USA), and were treated with $2 \%$ isoflurane during surgery and early reperfusion. A heating blanket was applied to the rats, in order to maintain a rectal temperature of $37.0 \pm 0.5^{\circ} \mathrm{C}$ throughout the experiments. A middle neck incision was made to expose the left common carotid artery (CCA). The external carotid artery was ligated using a bipolar coagulator (Hkpromed, Beijing, China). A nylon filament (diameter 0.24-0.28 mm) was gently inserted into the left internal carotid artery, until resistance was felt. Subsequently, the CBF dropped sharply, indicating that the ischemic model was successfully established. Reperfusion was induced by removing the filament after $90 \mathrm{~min}$ of ischemia. Sham-operated animals were subjected to the same anesthetic, incision and exposure of the vessels, without arterial occlusion. Ischemic postconditioning was established by repeatedly occluding and releasing the left CCA using aneurysm clips (cat. no. FE 681 K; Aesculap, Inc., Center Valley, PA, USA). In order to explore the combinative effects of early and delayed postconditioning, the two types of postconditioning were combined. The protocol for early plus delayed ischemic postconditioning is shown in Fig. 1A. Both types of postconditioning consisted of 10 cycles of 10 -sec reperfusion/10-sec occlusion; early postconditioning was conducted immediately following reperfusion, and late postconditioning took place $3 \mathrm{~h}$ after reperfusion.
CBF measurements. A laser Doppler flow meter (PeriFlux System 5000; Perimed AG, Järfälla, Sweden) was used to measure $\mathrm{CBF}$ throughout the experiments, and was connected to a standard laser Doppler monitor (PeriFlux 5000 Laser Doppler Perfusion Monitor and PeriFlux 5000 main unit; Perimed AG). The holder for the Doppler probe was fixed to the left side of the skull, in the region of the ischemic penumbra ( $2 \mathrm{~mm}$ lateral and $2 \mathrm{~mm}$ posterior to the bregma). The baseline value was regarded as the recorded $\mathrm{CBF}$ prior to occlusion. All $\mathrm{CBF}$ values were expressed as a percentage relative to the baseline.

Neurological behavioral assessment. A fully computerized electronically controlled rotarod treadmill for rats (Accuscan Instruments, Inc., Columbus, OH, USA) was used to investigate motor function. Each rat was placed in a neutral position on a cylinder with a diameter of 3.75 inches. The speed of the rod accelerated linearly between 0 and $24 \mathrm{rpm}$ within $60 \mathrm{sec}$. The time that rats maintained on the rotarod was recorded automatically. The maximum score given to an animal was fixed at $60 \mathrm{sec}$. All rats were given three trials and the average score from these three trials was calculated.

Infarct volume measurement. The rats were euthanized 3 days after surgery using $\mathrm{CO}_{2}$ and the brain tissue was harvested after transcardial perfusion with cold phosphate-buffered saline (PBS). Following 3 min of freezing, the brains were cut into six 2-mm coronal sections. The tissue sections were then immediately stained with $2 \%$ 2,3,5-triphenyl tetrazolium chloride (TTC) in PBS at $37^{\circ} \mathrm{C}$ for $20 \mathrm{~min}$. Images of the stained sections were captured using a digital camera (Nikon E5100; Nikon Corporation, Tokyo, Japan), and were then quantified using ImageJ version 1.37c software (National Institutes of Health, Bethesda, MA, USA). The infarct volume was the sum of the measured infarct areas of evenly sliced (2-mm) brain sections, according to Simpson's rule (14).

Brain edema measurement. The cerebral hemispheres, cerebellum and brain stem were separated. The wet weight was regarded as the immediate weight, and the dry weight was regarded as the weight after drying at $104^{\circ} \mathrm{C}$ for $48 \mathrm{~h}$. The water content percentage was determined using the following formula: [(wet weight-dry weight)/wet weight] x 100 .

In situ labeling of DNA fragmentation using TUNEL staining. In order to determine the level of apoptosis in the penumbral area, TUNEL staining was performed on each tissue section, according to the manufacturer's instructions (Roche Diagnostics GmbH, Mannheim, Germany). Briefly, the slides were washed with PBS at room temperature for $30 \mathrm{~min}$ and incubated with $0.1 \%$ Triton $\mathrm{X}-100$ in $0.1 \%$ sodium citrate solution at $4^{\circ} \mathrm{C}$ for $2 \mathrm{~min}$. Approximately $50 \mu \mathrm{l}$ TUNEL mixture solution was applied to each slide at $37^{\circ} \mathrm{C}$ for $1 \mathrm{~h}$. All of the sections were overlaid with prolonged mounting reagent and DAPI (Invitrogen Life Technologies, Carlsbad, CA, USA), and were covered with coverslips. The Nikon ECLIPSE Ti fluorescence microscope (Nikon Corporation) and CoolSNAP photometrics camera (Nikon Corporation) were used to visualize and capture the images. TUNEL-positive cells, indicated by bright green fluorescence, were quantified using 
the NIS-Elements BR diagnostic software version 3.2 (Nikon Corporation). The number of TUNEL-positive cells was counted from three random $1 \times 1 \mathrm{~mm}^{2}$ areas.

Immunofluorescence staining. Brain sections (10 $\mu \mathrm{m})$ were incubated with $10 \%$ normal goat serum/0.1\% Triton-X 100 in PBS blocking solution at room temperature for $1 \mathrm{~h}$. Slides were then incubated with the following corresponding primary antibodies: Polyclonal rabbit anti-rat BDNF (1:200; cat. no. ab6201; Epitomics, Inc., Burlingame, CA, USA), monoclonal anti-neuron-specific nuclear protein $(\mathrm{NeuN}$; 1:500; cat. no. MAB377B; EMD Millipore, Bedford, MA, USA) and monoclonal anti-glial fibrillary acidic protein (GFAP; 1:200; cat. no. G3893; Sigma-Aldrich, St. Louis, MO, USA), overnight at $4^{\circ} \mathrm{C}$. Following incubation, slides were then washed and incubated for $1 \mathrm{~h}$ with the following secondary antibodies: Alexa Fluor 488 goat anti-rabbit Immunoglobulin G (1:300; cat. no. A31267; Invitrogen Life Technologies, Camarillo, CA, USA) and Alexa Fluor 568 goat anti-rat (1:500; cat. no. A10042; Invitrogen Life Technologies). DAPI was used to stain the nuclei (Sigma-Aldrich). All of the slides were visualized under a Nikon ECLIPSE Ti fluorescence microscope, loaded with a CoolSNAP photometrics camera at 400x magnification.

Western blot analysis. The expression levels of CREB-ERK pathway-associated proteins were detected by western blot analysis. Protein extraction reagents (Sigma-Aldrich) were applied for total protein isolation and purification. Protein samples $(\sim 50 \mu \mathrm{g})$ were separated by $11.5 \%$ SDS-PAGE (Sigma-Aldrich). Polypeptides were electrophoretically transferred onto an Immobilon polyvinylidene fluoride membrane (EMD Millipore) at $300 \mathrm{~mA}$ for $1 \mathrm{~h}$ on ice. The membranes were then blocked with $5 \%$ non-fat milk in Tris-buffered saline with Tween (TBST). After blocking, the membranes were rinsed with PBS and incubated with the following antibodies: Anti-BDNF (1:500; Epitomics, Inc.), anti-phosphorylated (p) ERK1/2 (1:200; cat. no. 14227s; Cell Signaling Technology, Inc., Danvers, MA, USA), anti-p-CREB (1:600; cat. no. 8212s; Cell Signaling Technology, Inc.) and anti- $\beta$-actin (1:1,000; cat. no. A2022; Sigma-Aldrich) overnight at $4{ }^{\circ} \mathrm{C}$. The membranes were then washed with TBS and were treated with horseradish peroxidase-conjugated secondary antibodies (1:800; cat. no. sc-2448; Santa Cruz Biotechnology, Inc., Dallas, TX, USA) using 1\% non-fat milk in TBST for $2 \mathrm{~h}$ at room temperature. The blots were visualized using the ChemiDoc ${ }^{\mathrm{TM}}$ XRS Imaging system (Bio-Rad Laboratories, Inc., Hercules, CA, USA), and the band densities were quantified using the Multi Gauge Software of Science Lab 2006 (Fujifilm Corporation, Tokyo, Japan). Six animals were analyzed from each group for the western blot analyses.

Statistical analysis. Data are expressed as the mean \pm standard error of the mean. The neurological scores were analyzed using the Mann-Whitney non-parametric test. Statistical analysis was performed using analysis of variance, followed by Dunnett's post hoc test. $\mathrm{P}<0.05$ (95\% confidence interval) was considered to indicate a statistically significant difference. SPSS version 15.0 software (SPSS, Inc., Chicago, IL, USA) was used to conduct statistical analyses.

\section{Results}

Combinative ischemic postconditioning further reduces infarct volume, compared with early or late postconditioning alone, and attenuates behavioral deficits in MCAO rats. In order to determine the effects of the combination of early and delayed ischemic postconditioning on brain ischemic injury, the infarct size due to ischemic insult was measured. Representative images of TTC-stained brain sections from rats subjected to MCAO are shown in Fig. 1B. The rats in the sham group exhibited no cerebral infarcts. In the MCAO group, large infarcts developed, which were predominantly located in the cerebral cortex and striatum. The normalized infarct volume in the MCAO group was $28.16 \pm 1.79 \%$. Early and delayed ischemic postconditioning reduced the infarct volume, and the normalized infarct volumes in these groups were $9.38 \pm 0.96$ and $10.33 \pm 1.31 \%$, respectively. The combination of early and delayed ischemic postconditioning further reduced the infarct volume to $2.22 \pm 0.65 \%$, which was approximately $22 \%$ lower than that of the MCAO group $(\mathrm{P}<0.01), 7 \%$ lower than that of the IPe group $(\mathrm{P}<0.05)$ and $8 \%$ lower than that of the IPd group $(\mathrm{P}<0.05$, Fig. 1C).

The rotarod test was performed in order to test the neurological function of rats following ischemic injury. The results indicated that early, delayed and combinative ischemic postconditioning significantly attenuated the neurological deficits, compared with the MCAO group ( $\mathrm{P}<0.05$, Fig. 1D). However, no significant differences were detected among the three ischemic postconditioning groups.

Combinative ischemic postconditioning stabilizes changes in $\mathrm{CBF}$ and attenuates brain edema. During ischemia, $\mathrm{CBF}$ was decreased to equivalent extents $(51.64 \pm 3.08 \%$ of baseline) in all groups, apart from the sham group. Following reperfusion, a short period of hyperperfusion $(168.67 \pm 6.11 \%)$, which lasted $\sim 50 \mathrm{~min}$, followed by a long period of hypoperfusion, was observed in the MCAO group (Fig. 2A). Although early postconditioning did not shorten the time of hyperperfusion, the level of hyperperfusion was reduce to $138.67 \pm 3.28 \%$ in the IPe group ( $\mathrm{P}<0.05$ compared with the MCAO and IPd groups) and $132.87 \pm 3.31 \%$ in the IPc group $(\mathrm{P}<0.05$ compared with the MCAO and IPd groups). Following $\sim 3 \mathrm{~h}$ of hypoperfusion, delayed postconditioning increased the $\mathrm{CBF}$ value to $119.67 \pm 1.17 \%$ in the IPd group $(\mathrm{P}<0.05$ compared with the MCAO and IPe groups), and $122.17 \pm 2.74 \%$ in the IPc group $(\mathrm{P}<0.05$ compared with the MCAO and IPe groups). These results suggest that combinative ischemic postconditioning significantly attenuated early hyperperfusion following ischemic reperfusion, as well as the subsequent hypoperfusion.

The wet-dry method was used to assess the level of brain edema 3 days after MCAO. The left brain hemisphere $(84.36 \pm 0.72 \%)$ contained more water than the right brain hemisphere $(80.81 \pm 0.88 \%)$ in the MCAO group. Early, delayed and combinative ischemic postconditioning all significantly reduced the water content of the left brain hemisphere $(\mathrm{P}<0.05)$, compared with the MCAO group. However, no significant differences were detected among the three ischemic postconditioning groups (Fig. 2B).

Combinative ischemic postconditioning further inhibits apoptosis in the penumbral area compared with early or late 
A

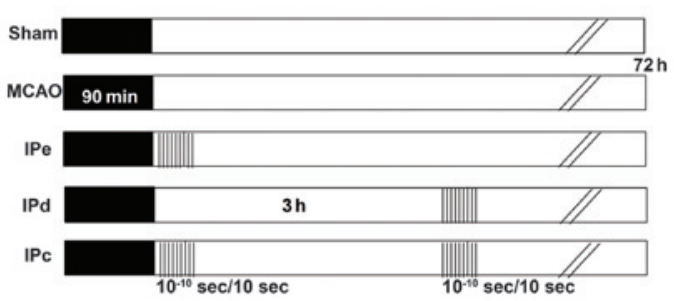

C

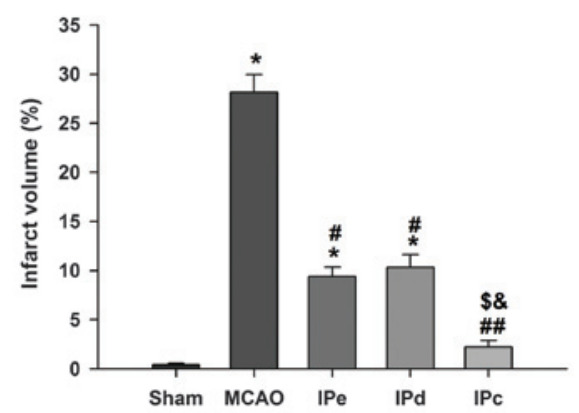

B

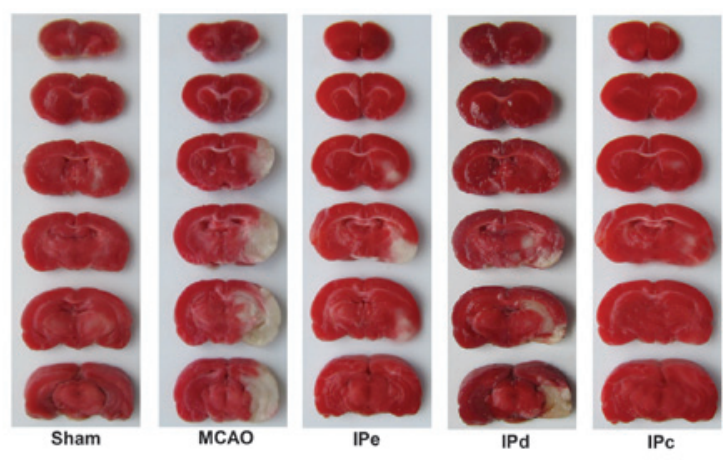

D

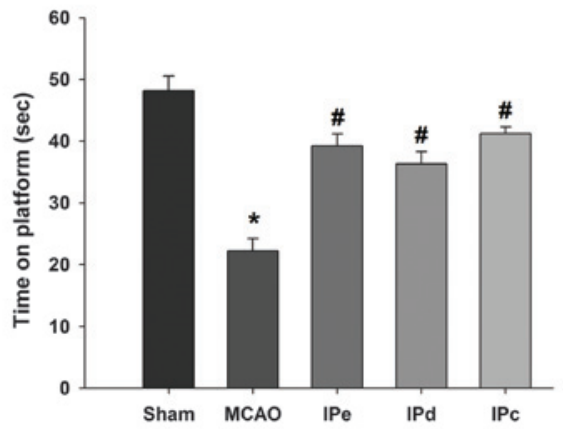

Figure 1. Combination of IPe and IPd reduced infarct volumes and neurological deficit in MCAO rats. (A) Protocol for IPc ( $\mathrm{n}=26$ ), in which postconditioning was conducted by occluding and releasing the ipsilateral common carotid artery. IPe $(=28)$ was performed by conducting $10 \mathrm{cycles}$ of $10 / 10$-sec reperfusion/re-occlusion immediately after MCAO. IPd $(\mathrm{n}=28)$ was performed $3 \mathrm{~h}$ after MCAO. Ischemic injury was induced by MCAO (MCAO, $\mathrm{n}=30)$. The sham group $(\mathrm{n}=24)$ was used as a control. (B) Representative infarct volume from each group, demonstrated by staining with 2,3,5-triphenyl tetrazolium chloride. (C) Average infarct volume in rats treated with postconditioning. (D) Ischemic postconditioning improved motor function, as evaluated by the rotarod test. Data are presented as the mean \pm standard error of the mean. ${ }^{*} \mathrm{P}<0.05$, compared with the sham group; ${ }^{\#} \mathrm{P}<0.05$ and ${ }^{\# \#} \mathrm{P}<0.01$, compared with the MCAO group; ${ }^{\$} \mathrm{P}<0.05$, compared with the IPe group; and ${ }^{\&} \mathrm{P}<0.05$, compared with the IPd group. MCAO, middle cerebral artery occlusion; IPe, early ischemic postconditioning; IPd, delayed ischemic postconditioning; IPC, combined IPe and IPd.

A

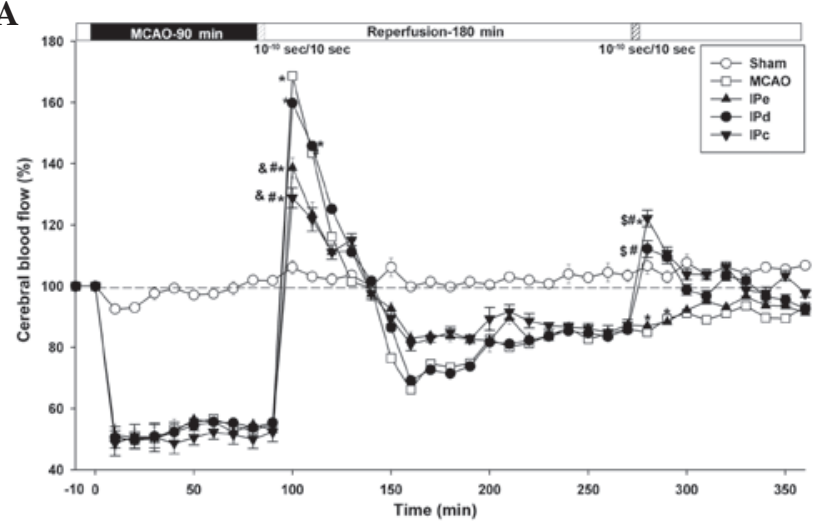

B

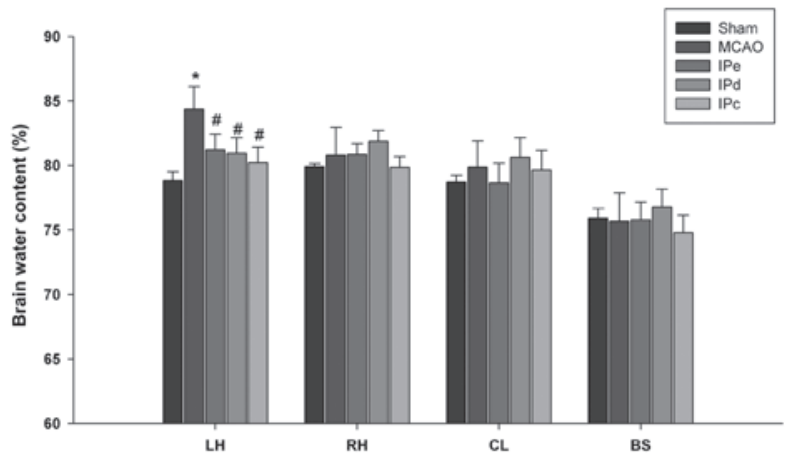

Figure 2. Combination of early and delayed postconditioning stabilized CBF and attenuated brain edema. (A) Changes in CBF in the MCA area following ischemia and ischemic postconditioning. Data are presented as the mean \pm standard error of the mean; $\mathrm{n}=24$. ${ }^{*} \mathrm{P}<0.05, \mathrm{compared}$ with the sham group; ${ }^{\#} \mathrm{P}<0.05$, compared with the MCAO group; ${ }^{\$} \mathrm{P}<0.05$, compared with the IPe group; and ${ }^{\&} \mathrm{P}<0.05$, compared with the IPd group. (B) Brain water content of the LH, RH, $\mathrm{CL}$ and $\mathrm{BS}$ following ischemia and ischemic postconditioning. Data are presented as the mean \pm standard error of the mean; $\mathrm{n}=6$. * $\mathrm{P}<0.05$, compared with the sham group; and " $\mathrm{P}<0.05$ compared with the MCAO group. $\mathrm{CBF}$, cerebral blood flow; MCAO, middle cerebral artery occlusion; IPe, early ischemic postconditioning; IPd, delayed ischemic postconditioning; IPc, combinative ischemic postconditioning; LH, left hemisphere; RH, right hemisphere; CL, cerebellum; BS, brain stem.

postconditioning alone. In pathological and anatomical terms, the penumbra is an area surrounding a severely ischemic core. This area is where pharmacological interventions are most likely to attenuate the neurological injury and improve clinical outcome (Fig. 3A). TUNEL-positive staining was absent in the sham-treated group (Fig. 3B). Numerous bright green dots, which indicate the presence of TUNEL-positive cells, were observed in the MCAO group (Fig. 3C). There were few TUNEL-positive cells observed among rats that were administered with early, delayed and combinative ischemic postconditioning treatment (Fig. 3D-F). Quantitative analysis indicated that the number of TUNEL-positive cells in the MCAO group was 409.78 \pm 45.23 . Early and delayed ischemic postconditioning significantly reduced the level of 

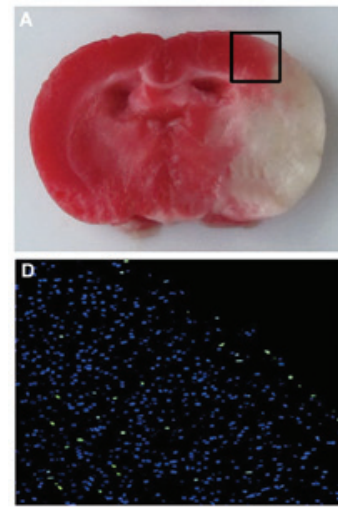
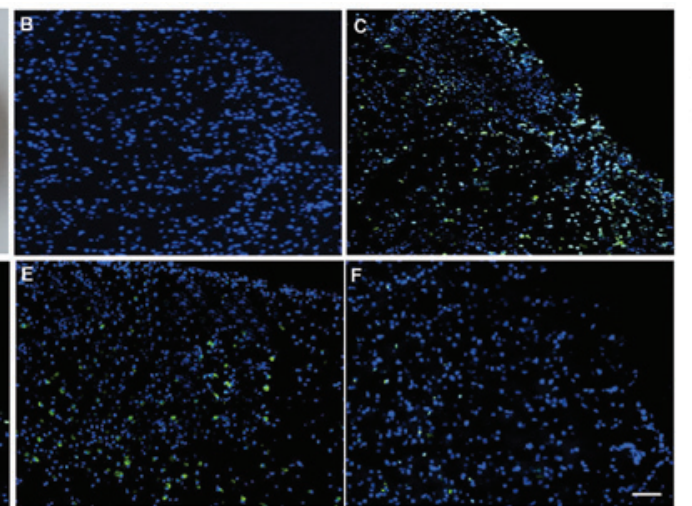

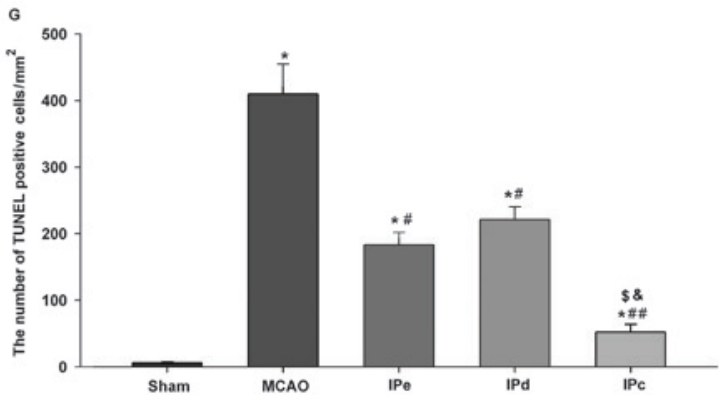

Figure 3. Combination of IPe and IPd inhibited the levels of apoptosis in the penumbral area. (A) The black square indicates the penumbral area Immunofluorescence results of the TUNEL assay in the (B) sham, (C) MCAO, (D) IPe, (E) IPd and (F) IPc groups. Bright green dots indicate TUNEL-positive apoptotic cells. Scale bar represents $50 \mu \mathrm{m}$. (G) Analysis of apoptotic cells 3 days after MCAO. TUNEL-positive cells were counted from three random $1 \mathrm{x} 1 \mathrm{~mm}^{2}$ areas. Data are presented as the mean \pm standard error of the mean; $\mathrm{n}=9$ per group. ${ }^{*} \mathrm{P}<0.05$, compared with the sham group; ${ }^{\#} \mathrm{P}<0.05$ or ${ }^{\# \#} \mathrm{P}<0.01$, compared with the MCAO group; ${ }^{\$} \mathrm{P}<0.05$, compared with the IPe group; and ${ }^{\&} \mathrm{P}<0.05$, compared with the IPd group. MCAO, middle cerebral artery occlusion; IPe, early ischemic postconditioning; IPd, delayed ischemic postconditioning; IPc, combinative ischemic postconditioning.
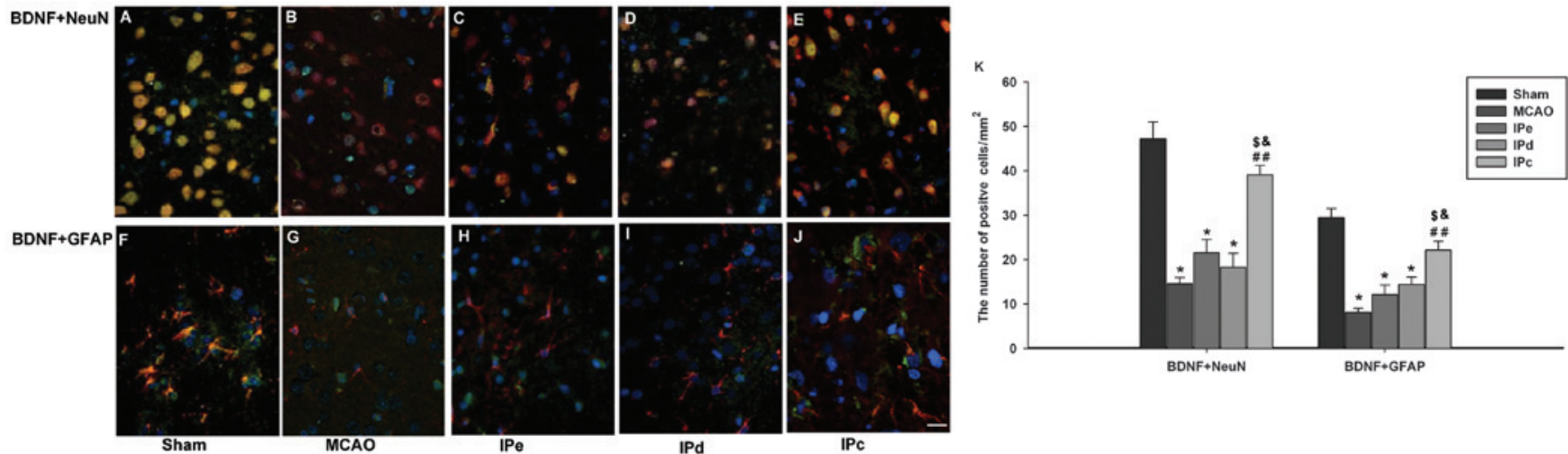

Figure 4. Combination of IPe and IPd increased the expression of BDNF in the penumbral area. Coronal sections were stained with the following specific antibodies 3 days after the operation: BDNF (green), with either NeuN (red) or GFAP (red). Mounting medium contained DAPI, which counterstained the nuclei blue. Merged pictures, which co-expressed (A)-(E) NeuN and BDNF or (F)-(J) GFAP and BDNF, were shown with yellow. Scale bar indicates 100 $\mu$ m. (K) Quantitative analysis of BDNF-positive cells were analyzed by ImageJ software. Data are presented as the mean \pm standard error of the mean; $\mathrm{n}=9$ per group. ${ }^{*} \mathrm{P}<0.05$, compared with the sham group; ${ }^{\# /} \mathrm{P}<0.01$, compared with the MCAO group; ${ }^{\$} \mathrm{P}<0.05$, compared with the IPe group; and \& $\mathrm{P}<0.05$, compared with the IPd group. BDNF, brain-derived neurotrophic factor; GFAP, glial acidic fibrillary protein; NeuN, neuron-specific nuclear protein; MCAO, middle cerebral artery occlusion; IPe, early ischemic postconditioning; IPd, delayed ischemic postconditioning; IPc, combinative ischemic postconditioning.

apoptosis, compared with the MCAO group ( $\mathrm{P}<0.05$, Fig. 3G). Combinative ischemic postconditioning further decreased the rate of apoptosis following ischemia in the penumbral area $(\mathrm{P}<0.01$, compared with the MCAO group; $\mathrm{P}<0.05$, compared with the IPe and IPd groups; Fig. 3G).

Combinative ischemic postconditioning increases the expression levels of BDNF in the penumbral area. The present study sought to determine whether ischemic postconditioning induces BDNF in the penumbral area, using immunofluorescence staining. Neurons in the sham control group exhibited BDNF-positive immunoreactivity, as assessed by BDNF+NeuN double staining (Fig. 4A). Astrocytes in this groups were also positive for BDNF, as assessed by BDNF+GFAP double staining (Fig. 4F). BDNF immunoreactivity was significantly lower in the MCAO group, compared with the sham group (Fig. 4B and G). Neither early nor delayed ischemic postconditioning alone had a significant effect on expression of BDNF (Fig. 4C, D, H and I). However, BDNF immunoreactivity was higher in the combinative ischemic postconditioning group, compared with that in the MCAO, IPe and IPd groups (Fig. 4E and $G$ ). Quantitative analysis also indicated that BDNF-positive signals were higher in the IPc group, compared with those in the MCAO ( $\mathrm{P}<0.01)$, IPe $(\mathrm{P}<0.05)$ and IPd groups $(\mathrm{P}<0.05$; Fig. 4K).

Combinative ischemic postconditioning upregulates BDNF protein expression levels by phosphorylating CREB and $E R K 1 / 2$. Western blot analyses indicated that combinative ischemic postconditioning increased the protein expression levels of BDNF in the penumbral area (Fig. 5A). Western blot analysis was also performed to investigate whether combinative ischemic postconditioning results in the phosphorylation of CREB and ERK1/2 within the cortex. No significant differences in the protein expression of total CREB and ERK1/2 were detected among the groups. However, administration of combinative ischemic postconditioning significantly increased the expression of $\mathrm{p}-\mathrm{CREB}$, compared with that of the MCAO $(\mathrm{P}<0.01)$, IPe $(\mathrm{P}<0.05)$ and IPd groups $(\mathrm{P}<0.05$; Fig. 5A and $\mathrm{B})$. Similar results 


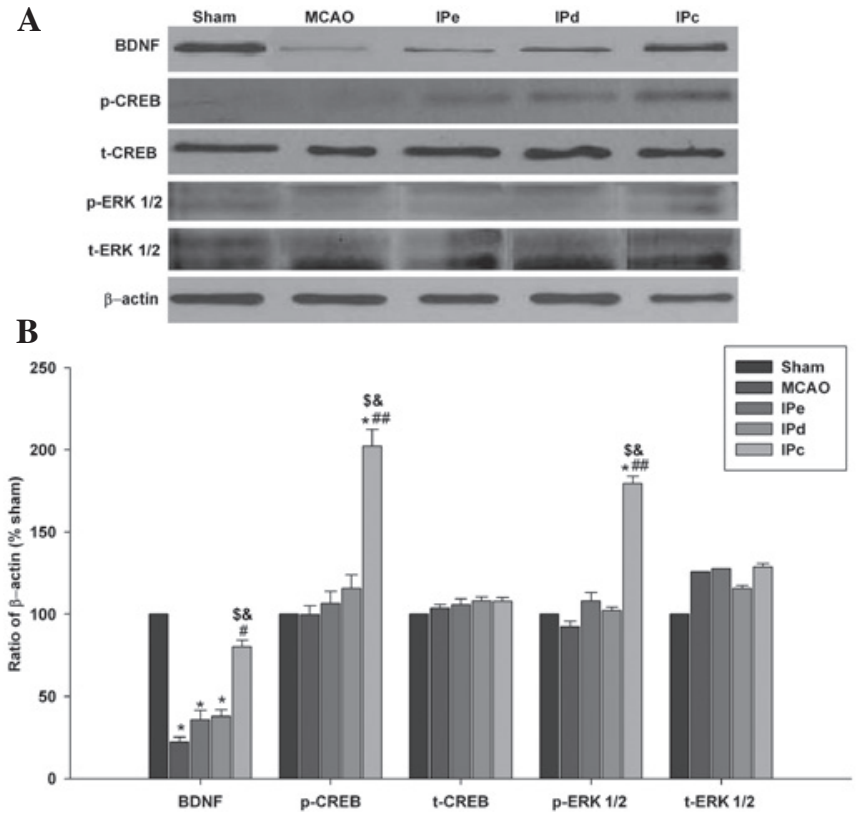

Figure 5. Combination of IPe and IPd upregulated the protein expression levels of BDNF, p-CREB and p-ERK1/2. (A) Protein expression levels of BDNF, p-CREB, t-CREB, p-ERK1/2 and t-ERK1/2 in the sham, MCAO, IPe, IPd and IPc rats. (B) Optical density analysis of BDNF, p-CREB, t-CREB, p-ERK1/2 and $t-E R K 1 / 2$ expression. Data are presented as the mean \pm standard error of the mean; $\mathrm{n}=9$ per group. ${ }^{*} \mathrm{P}<0.05$, compared with the sham group; ${ }^{*} \mathrm{P}<0.05$ or ${ }^{\# \#} \mathrm{P}<0.01$, compared with the MCAO group; ${ }^{\$} \mathrm{P}<0.05$ compared with the IPe group; and ${ }^{\circledR} \mathrm{P}<0.05$, compared with the IPd group. BDNF, brain-derived neurotrophic factor; $p$, phosphorylated; $t$, total; CREB, cAMP response element-binding protein; ERK 1/2, extracellular signal-regulated kinases $1 / 2$; MCAO, middle cerebral artery occlusion; IPe, early ischemic postconditioning; IPd, delayed ischemic postconditioning; IPc, combinative ischemic postconditioning.

were obtained for the expression of p-ERK1/2 (Fig. 5A and B), which is the upstream phosphorylating enzyme of CREB.

\section{Discussion}

The results of the present study demonstrated that the combination of early and delayed ischemic postconditioning reduced infarct volume and stabilized CBF disturbance in rats, following focal brain ischemia, compared with no treatment, as well as with early or late postconditioning treatment alone. The potential neuroprotective mechanisms underlying the protective effect of combinative ischemic postconditioning against focal ischemic injury may involve the reduction of neuronal apoptosis in the penumbral area. Furthermore, it was demonstrated that combinative ischemic postconditioning upregulated the expression of BDNF, which aids the repair of neuronal injury in the central nervous system. In addition, the majority of BDNF-positive cells were detected by staining with either anti-NeuN or anti-GFAP. Combinative ischemic postconditioning also activated ERK1/2 and CREB in the cortex, following focal ischemia. These results suggested that combinative ischemic postconditioning induces BDNF expression in neurons and astrocytes, and protects against neurological injury following brain ischemia, which may be mediated by the phosphorylation of ERK1/2 and CREB.

It has been established that numerous neuroprotectants, which are highly effective in animal models of stroke, fail in clinical trials due to side effects, which frequently result in premature termination of a trial (e.g., MK-801, ZK200755) (15-17). Previous studies have aimed to overcome this limitation by developing methods that induce, mimic or enhance endogenous protective responses, and do not interfere with physiological neurotransmission (18-20). Therefore, endogenous neuroprotection is a novel strategy that has been increasingly focused on within stroke research (21).

Ischemic conditioning has been suggested as a useful method with which to generate ischemic tolerance or endogenous neuroprotection (20). There are two types of ischemic conditioning: Ischemic preconditioning and ischemic postconditioning. Ischemic preconditioning may be induced by the application of transient episodes of nonlethal ischemia prior to stroke (22). Ischemic postconditioning is defined as numerous repeated cycles of brief reperfusion and re-occlusion, which may protect against lethal ischemic injury and subsequent reperfusion damage (23). Currently, both of these types of ischemic conditioning are hypothesized to be possible therapeutic strategies. However, the translational application of ischemic preconditioning is hindered by the fact that it can only be applied for strokes or ischemic injuries that may be predicted $(24,25)$. Therefore, ischemic postconditioning, which can be applied after ischemia, may be a better approach.

Recently, it has been demonstrated that two types of postconditioning, early ischemic postconditioning (26) and delayed ischemic postconditioning (23), exhibit neuroprotective effects, and may reduce neuronal injury following brain ischemia. However, there are few reports regarding the neuroprotective effects of a combination of these two types of postconditioning. Therefore, the aim of the present study was to evaluate the effects of combined early and delayed ischemic postconditioning. In the present study, early ischemic postconditioning or delayed ischemic postconditioning each reduced the infarct volume following brain ischemia. However, combinative ischemic postconditioning further reduced infarct volume $(\mathrm{P}<0.05)$, compared with either approach alone. Disturbances to $\mathrm{CBF}$ occur throughout the period of reperfusion. Following reperfusion, there is a short period of hyperperfusion, followed by a longer period of hypoperfusion. The results of the present study indicated that combinative ischemic postconditioning may stabilize $\mathrm{CBF}$ disturbances during the early hyperperfusion and later hypoperfusion periods.

BDNF is considered an important member of the neurotrophin family, which contributes to the maintenance and survival of neurons (27), and stimulates the differentiation and growth of new neurons and synapses (28). The primary role of BDNF is associated with increased long-term potentiation and neurogenesis (29). Numerous studies have shown that BDNF decreases infarct volume and improves neurological outcomes, when exogenously administered (30) or when overexpressed using genetic methods in vivo (31) in experimental models of stroke. However, the mechanisms underlying the effects of ischemic postconditioning on the production of BDNF remain unclear. The present study used immunofluorescence staining and western blot analysis to detect BDNF expression in the brain penumbral area following focal brain ischemia. The results indicated that neither early nor 
delayed ischemic postconditioning significantly increased the expression levels of BDNF. However, combinative ischemic postconditioning upregulated the expression levels of BDNF in neuronal cells and astrocytes.

Additional mechanisms of combinative ischemic postconditioning were hypothesized to involve CREB, a transcription factor of BDNF. Furthermore, ERK1/2, which is the upstream phosphorylating enzyme of CREB, activates and phosphorylates CREB at Ser133 (32), resulting in the upregulation of pro-survival CREB target genes, including $\operatorname{BDNF}(33,34)$. Therefore, in the present study western blotting was used to detect the protein expression levels of CREB and ERK1/2. The results demonstrated that no difference in the expression of total CREB and ERK1/2 protein among the groups. However, combinative ischemic postconditioning significantly increased the protein expression levels of $\mathrm{p}-\mathrm{CREB}$ and $\mathrm{p}-\mathrm{ERK} 1 / 2$ in the penumbral area following focal brain ischemia.

The results of the present study demonstrated that a combination of early and delayed ischemic postconditioning had stronger neuroprotective effects on focal brain ischemia, compared with early or delayed ischemic postconditioning alone. This effect may be associated with the stabilization of CBF disturbances and a reduction in apoptosis in the penumbral area following focal brain ischemia. Furthermore, it was indicated that combinative ischemic postconditioning upregulated the expression of BDNF, in neurons and astrocytes, and protected against neurological damage following brain ischemic injury. These effects may be associated with activation of ERK1/2 and CREB. Although there were some limitations to the present study, such as the use of only one model of combinative postconditioning and the fact that the neuroprotective effects were examined using only a rat model, combinative ischemic postconditioning appeared to alleviate or even prevent ischemic brain injury. Therefore, combinative ischemic postconditioning has the potential for future clinical application and requires further investigation.

\section{Acknowledgements}

The present study was supported by the National Nature Science Foundation (grant no. 81000498).

\section{References}

1. Blanco M and Castillo J: Stroke in 2012: Major advances in the treatment of stroke. Nat Rev Neurol 9: 68-70, 2013.

2. Liu J, Xu Q, Wang H, et al: Neuroprotection of ischemic postconditioning by downregulating the postsynaptic signaling mediated by kainate receptors. Stroke 44: 2031-2035, 2013.

3. Sandu N and Schaller B: Postconditioning: A new or old option after ischemic stroke? Expert Rev Cardiovasc Ther 8: 479-482, 2010.

4. Zhao H: Ischemic postconditioning as a novel avenue to protect against brain injury after stroke. J Cereb Blood Flow Metab 29: 873-885, 2009

5. Danielisova V, Burda J, Nemethova M, et al: An effective combination of two different methods of postconditioning. Neurochem Res 37: 2085-2091, 2012.

6. Zhao H, Ren C, Chen X and Shen J: From rapid to delayed and remote postconditioning: The evolving concept of ischemic postconditioning in brain ischemia. Curr Drug Targets 13: 173-187, 2012.

7. Dauncey MJ: Recent advances in nutrition, genes and brain health. Proc Nutr Soc 71: 581-591, 2012.
8. Lu B, Nagappan G, Guan X, et al: BDNF-based synaptic repair as a disease-modifying strategy for neurodegenerative diseases. Nat Rev Neurosci 14: 401-416, 2013.

9. Melo CV, Okumoto S, Gomes JR, et al: Spatiotemporal resolution of BDNF neuroprotection against glutamate excitotoxicity in cultured hippocampal neurons. Neuroscience 237: 66-86, 2013.

10. Singh M and Su C: Progesterone, brain-derived neurotrophic factor and neuroprotection. Neuroscience 239: 84-91, 2013.

11. Alboni S, Tascedda F, Corsini D, et al: Stress induces altered CRE/CREB pathway activity and BDNF expression in the hippocampus of glucocorticoid receptor-impaired mice. Neuropharmacology 60: 1337-1346, 2011.

12. Pardon MC, Roberts RE, Marsden CA, et al: Social threat and novel cage stress-induced sustained extracellular-regulated kinase1/2 (ERK1/2) phosphorylation but differential modulation of brain-derived neurotrophic factor (BDNF) expression in the hippocampus of NMRI mice. Neuroscience 132: 561-574, 2005.

13. Yifeng M, Bin W, Weiqiao Z, et al: Neuroprotective effect of sophocarpine against transient focal cerebral ischemia via down-regulation of the acid-sensing ion channel 1 in rats. Brain Res 1382: 245-251, 2011.

14. Thiele H, Paetsch I, Schnackenburg B, et al: Improved accuracy of quantitative assessment of left ventricular volume and ejection fraction by geometric models with steady-state free precession. J Cardiovasc Magn Reson 4: 327-339, 2002.

15. Diener HC, Lees KR, Lyden P, et al; SAINT I and II Investigators: NXY-059 for the treatment of acute stroke: Pooled analysis of the SAINT I and II Trials. Stroke 39: 1751-1758, 2008.

16. Furlan AJ: Challenges in acute ischemic stroke clinical trials. Curr Cardiol Rep 14: 761-766, 2012.

17. Shuaib A, Lees KR, Lyden P, et al; SAINT II Trial Investigators: NXY-059 for the treatment of acute ischemic stroke. N Engl J Med 357: 562-571, 2007.

18. Ducruet AF, Zacharia BE, Sosunov SA, et al: Complement inhibition promotes endogenous neurogenesis and sustained anti-inflammatory neuroprotection following reperfused stroke. PloS One 7: e38664, 2012.

19. Iadecola $C$ and Anrather J: Stroke research at a crossroad: Asking the brain for directions. Nat Neurosci 14: 1363-1368, 2011.

20. Ding ZM, Wu B, Zhang WQ, et al: Neuroprotective effects of ischemic preconditioning and postconditioning on global brain ischemia in rats through the same effect on inhibition of apoptosis. Int J Mol Sci 13: 6089-6101, 2012.

21. Wang Y, Reis C, Applegate R, et al: Ischemic conditioning-induced endogenous brain protection: Applications pre-, per- or post-stroke. Exp Neurol 15: 123-125, 2015.

22. Liu XQ, Sheng R and Qin Z: The neuroprotective mechanism of brain ischemic preconditioning. Acta Pharmacol Sin 30: 1071-1080, 2009.

23. Ren C, Gao X, Niu G, et al: Delayed postconditioning protects against focal ischemic brain injury in rats. PloS One 3: e3851, 2008.

24. Wang JY, Shen J, Gao Q, et al: Ischemic postconditioning protects against global cerebral ischemia/reperfusion-induced injury in rats. Stroke 39: 983-990, 2008.

25. Xing B, Chen $\mathrm{H}$, Zhang $\mathrm{M}$, et al: Ischemic postconditioning inhibits apoptosis after focal cerebral ischemia/reperfusion injury in the rat. Stroke 39: 2362-2369, 2008.

26. Liu C, Weaver J and Liu KJ: Early conditioning with oxygen oscillation: Neuroprotection by intermittent normobaric hyperoxia after transient focal cerebral ischemia in rats. Stroke 43: 220-226, 2012.

27. Stahl K, Mylonakou MN, Skare Ø, et al: Cytoprotective effects of growth factors: BDNF more potent than GDNF in an organotypic culture model of Parkinson's disease. Brain Res 1378: 105-118, 2011.

28. Baldelli P, Novara M, Carabelli V, et al: BDNF up-regulates evoked GABAergic transmission in developing hippocampus by potentiating presynaptic $\mathrm{N}^{-}$and $\mathrm{P} / \mathrm{Q}$-type $\mathrm{Ca}^{2+}$ channels signalling. Eur J Neurosci 16: 2297-2310, 2002.

29. Babu H, Ramirez-Rodriguez G, Fabel K, et al: Synaptic network activity induces neuronal differentiation of adult hippocampal precursor cells through BDNF signaling. Front Neurosci 3: 49, 2009.

30. Hyacinth HI, Gee BE, Adamkiewicz TV, et al: Plasma BDNF and PDGF-AA levels are associated with high TCD velocity and stroke in children with sickle cell anemia. Cytokine 60: 302-308, 2012. 
31. Chang DJ, Lee N, Choi C, et al: Therapeutic effect of BDNF-overexpressing human neural stem cells (HB1.F3.BDNF) in a rodent model of middle cerebral artery occlusion. Cell Transplant 22: 1441-1452, 2013.

32. Cao G, Zhu J, Zhong Q, et al: Distinct roles of methamphetamine in modulating spatial memory consolidation, retrieval, reconsolidation and the accompanying changes of ERK and CREB activation in hippocampus and prefrontal cortex. Neuropharmacology 67: 144-154, 2013.
33. Chen DY, Bambah-Mukku D, Pollonini G and Alberini CM: Glucocorticoid receptors recruit the CaMKII $\alpha$-BDNF-CREB pathways to mediate memory consolidation. Nat Neurosci 15: 1707-1714, 2012

34. Lesiak A, Pelz C, Ando H, et al: A genome-wide screen of CREB occupancy identifies the RhoA inhibitors Par6C and Rnd3 as regulators of BDNF-induced synaptogenesis. PloS One 8: e64658, 2013. 